\title{
SEMANTIC BORROWING FOR GENERALIZED ZERO-SHOT LEARNING
}

\author{
Xiao-wei CHEN
}

Sun Yat-sen University

\begin{abstract}
Generalized zero-shot learning (GZSL) is one of the most realistic problems, but also one of the most challenging problems due to the partiality of the classifier to supervised classes. Instance-borrowing methods and synthesizing methods solve this problem to some extent with the help of testing semantics, but therefore neither can be used under the class-inductive instance-inductive (CIII) training setting where testing data are not available, and the latter require the training process of a classifier after generating examples. In contrast, a novel method called Semantic Borrowing for improving GZSL methods with compatibility metric learning under CIII is proposed in this paper. It borrows similar semantics in the training set, so that the classifier can model the relationship between the semantics of zero-shot and supervised classes more accurately during training. In practice, the information of semantics of unseen or unknown classes would not be available for training while this approach does NOT need any information of semantics of unseen or unknown classes. The experimental results on representative GZSL benchmark datasets show that it can reduce the partiality of the classifier to supervised classes and improve the performance of generalized zero-shot classification.
\end{abstract}

Index Terms - Classification, generalized zero-shot learning, semantic borrowing

\section{INTRODUCTION}

Classification has made great progress driven by the advancement of deep learning, but a large number of instances for each class are required for training the model of the classifier with supervised learning. It is not always possible to label a large number of instances for each class in the real world. For example, the number of image instances of each class follows a long-tail distribution [1]. Furthermore, the classifiers trained on the instances for training cannot classify instances of the classes that the previous instances don't belong to, but these classifiers are often required to deal with instances of new classes without training from scratch in practical applications. These challenges severely limit the application of these classification methods in practice. Many methods have been proposed to overcome these difficulties [2], including open world recognition, open set recognition, cumulative learning, classincremental learning, few-shot learning, one-shot learning, zeroshot learning [3-5] and generalized zero-shot learning (GZSL) [67]. GZSL is to model novel classes without instances for training, distinguishing them from classes with instances for training. It is realistic, but it is also challenging.

In GZSL, seen classes refer to classes with training examples, while unseen classes refer to classes without training examples. The goal of GZSL is to learn a classifier on the training examples of seen classes, and then use it to predict class labels of testing examples of unseen or seen classes, where the testing examples of

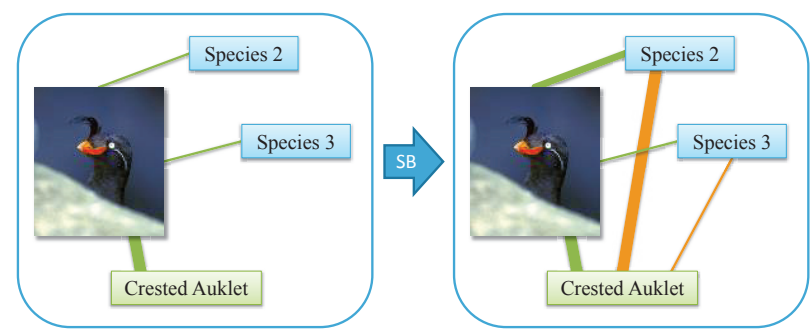

Fig. 1. Illustration of the improvement of compatibilities by SB. The thickness of the line between a photo and a text indicates the compatibility between them while the thickness of the line between two texts indicates the semantic similarity between them. By borrowing similar semantics in the training set, we can enable a classifier to model more accurately the relationship between the semantics of unseen and seen classes during training without the semantics of unseen classes.

seen classes are not in the training examples. The semantics of the label of a class can be defined by training examples of the class in traditional classification problems, but different from it, the semantics of the label of an unseen class cannot be defined by training examples in GZSL. To solve this problem, a semantic space can be defined, in which each label of a seen or unseen class is identified uniquely. The space can be constructed in different ways [2], by defining manually [3-5, 8-9], for example, defining some attributes, and then use their values to describe the semantics related to the features of the examples, or obtaining through learning [9-12], for example, learning the embedding of labels. After defining a semantic space, the GZSL problem becomes to model the relationship between the example feature space and the semantic space. According to whether the testing example features in the feature space and the testing semantics in the semantic space are available during training, there are three training settings for a GZSL classifier, namely class-inductive instance-inductive (CIII) setting, class-transductive instance-inductive (CTII) setting and class-transductive instance-transductive (CTIT) setting. CTII allows the use of testing semantics during training, CTIT also allows the use of unlabeled testing example features, and CIII allows neither of these two. Their further descriptions can be found in [2]. For different semantic spaces and different training settings, there have been proposed methods that can be used to solve GZSL problems, which can be divided into 6 groups [2], namely correspondence methods [9-10, 13-14], relationship methods [1516], combination methods [3], projection methods [11, 17-18], instance-borrowing methods [19] and synthesizing methods [2021]. Instance-borrowing methods borrow training examples based on the testing semantics to train the classifier, while synthesizing methods train generation models such as VAE and GAN to generate training examples for unseen classes based on the testing semantics to train the classifier. 
Due to the differences in the distributions of the seen and unseen classes, a GZSL classifier will suffer from the domain shift problem [22]. If a testing example may belong to a seen or unseen class, the classifier will be biased towards the seen class when classifying. Domain shift reduces the accuracy of generalized zeroshot learning [6]. Instance-borrowing methods and synthesizing methods solve this problem to some extent with the help of testing semantics, but therefore neither of them can be used under CIII [2] where testing data are invisible, and the latter always require the training process of a classifier after generating examples based on testing semantics.

In this paper, a method is proposed to improve the compatibility metric learning used in GZSL methods under CIII. In the GZSL methods based on compatibility metric learning, the relationship between features and semantics, that is, compatibility, is learned through metric learning, and then the differences among the compatibilities between a testing feature and all semantic candidates in this metric space are determined, and finally, the semantic candidate corresponding to the testing example feature is determined accordingly, so that the class label of the testing feature can be obtained, thus achieving the goal of GZSL. Different from the process above, by borrowing similar semantics in the training set, we can enable a classifier to model the relationship between the semantics of unseen and seen classes more accurately during training without the semantics of unseen classes, thereby reducing the partiality of the classifier to seen classes during testing to deal with the domain shift problem, as shown in Fig. 1. The proposed method is named Semantic Borrowing (SB).

The main contribution of this paper is $\mathrm{SB}$, which can improve GZSL methods with compatibility metric learning without surpassing CIII. In practice, the information of semantics of unseen or unknown classes would not be available for training while this approach does NOT need any information of semantics of unseen or unknown classes. Experiments also verified this under the GZSL evaluation protocol [7].

\section{METHOD}

SB is based on the compatibility metric learning in GZSL methods. As mentioned earlier, GZSL with compatibility metric learning will learn the compatibilities between features and semantics through metric learning. At the same time, SB learns additionally the compatibility between each feature and the most similar semantic vector to the semantic vector corresponding to the feature. With the help of this information, the differences among the compatibilities between a testing feature and all semantic candidates in the learned metric space will be more accurate. In other words, the relationship between the semantics of unseen and seen classes is modeled more accurately by the classifier. SB is illustrated in the right panel of Fig. 1.

The set of all seen classes is denoted as $B_{S}$ and the set of all unseen classes $B_{u}, B_{s} \cap B_{u}=\varnothing$, then the set of all classes $B=$ $B_{S} \cup B_{u}$. For any class $b \in B$, there is a unique corresponding semantic vector $s \in \mathbb{R}^{n}$. The set of all semantic vectors is denoted as $S$, and the set of all semantic vectors of seen classes $S_{S}$, then the set of all seen class examples $D_{s}=\left\{(f, s) \mid f \in F_{s}, s \in S_{s}\right\}$, where $F_{S} \subset F \subseteq \mathbb{R}^{m}$ is the set of all features of seen class examples, and $F$ is the set of all features of examples. The set of all unseen examples is denoted as $D_{u}$, then GZSL learns a classifier on the training set $D_{t r}=\left\{(f, s) \mid f \in F_{t r}, s \in S_{t r}\right\} \subset D_{s}$ to obtain the classes of example features in testing sets $D_{t e-s} \subset D_{s}$ and $D_{u}$, where $D_{t r} \cap D_{t e-s}=\emptyset$.

\subsection{Framework}

The compatibilities between features and semantics form a metric space in which the compatibility between a feature and its corresponding semantic vector will be greater than that between the feature and other semantics. In order to learn such a space, we can use a linear model or a nonlinear model to fit it, but because they have different fitting capabilities due to the different complexities of a linear model and a nonlinear model, we need define different objectives to train them.

For the linear model, in order to adapt to its limited fitting ability, we can train a compatibility function $c: F \times S \rightarrow \mathbb{R}$ on the training dataset with the objective of symmetric structured joint embedding in the previous multi-modal structured learning methods $[8-9,12]$ :

$$
L_{1}\left(f_{i}, s_{i} ; \theta\right)=L_{f}\left(f_{i}, s_{i} ; \theta\right)+L_{s}\left(f_{i}, s_{i} ; \theta\right)
$$

where $\left(f_{i}, s_{i}\right) \in D_{t r}$ and the two misclassification losses are:

$$
\begin{aligned}
& L_{f}\left(f_{i}, s_{i} ; \theta\right) \\
& =\frac{1}{\left|S_{t r}\right|-1} \sum_{s \in S_{t r} \backslash\left\{s_{i}\right\}} \max \left\{0,1+c\left(f_{i}, s ; \theta\right)-c\left(f_{i}, s_{i} ; \theta\right)\right\} \\
& L_{s}\left(f_{i}, s_{i} ; \theta\right) \\
& =\frac{1}{\left|F_{t r}\right|-1} \sum_{f \in F_{t r} \backslash\left\{f_{i}\right\}} \max \left\{0,1+c\left(f, s_{i} ; \theta\right)-c\left(f_{i}, s_{i} ; \theta\right)\right\}
\end{aligned}
$$

where $|\cdot|$ indicates the cardinality of a set, $B \backslash A$ denotes the relative complement of $A$ in $B$.

However, in order for the classifier to model the relationship between the semantics of unseen and seen classes more accurately during training, we can add a new objective that borrows similar semantics in the training set:

$$
\begin{aligned}
& L_{2}\left(f_{i}, s_{i}, s_{j} ; \theta\right) \\
& =\frac{\sum_{s \in S_{t r} \backslash\left\{s_{i}, s_{j}\right\}} \max \left\{0,1+c\left(f_{i}, s ; \theta\right)-c\left(f_{i}, s_{j} ; \theta\right)\right\}}{\left|S_{t r}\right|-\left|\left\{s_{i}, s_{j}\right\}\right|}
\end{aligned}
$$

where $s_{j} \in S_{t r}$ is the most similar semantic vector to $s_{i}$ in the training set.

Finally, the overall loss for a linear model is:

$$
\begin{aligned}
L(\theta) & =\sum_{\substack{\left(f_{i}, s_{i}\right) \in D_{t r}\\
}} L_{1}\left(f_{i}, s_{i} ; \theta\right) \\
& \alpha \sum_{\substack{\left(f_{i}, s_{i}\right) \in D_{t r} \\
s_{j}=C\left(s_{i}\right)}} L_{2}\left(f_{i}, s_{i}, s_{j} ; \theta\right)+\beta\|\theta\|_{2}
\end{aligned}
$$

where $\alpha \in(0,1), \beta$ controls weight decay, $C: S_{t r} \rightarrow S_{t r}$ is used to find similar semantics. By minimizing this loss, we can make the compatibility between a feature and the most semantically similar semantic vector to it also greater than that between the feature and other semantics.

For the nonlinear model, because of its strong fitting ability, we can use the MSE loss to train a compatibility function on the training set as in [23]. Therefore, $L_{1}$ in Eq. 5 is changed into: 


\begin{tabular}{l|cccccc}
\hline Dataset & $n$ & $\left|B_{s}\right|$ & $\left|B_{u}\right|$ & $\left|D_{t r}\right|$ & $\left|D_{t e-s}\right|$ & $\left|D_{u}\right|$ \\
\hline \hline CUB [24] & 312 & 150 & 50 & 7057 & 1764 & 2967 \\
AWA1 [3] & 85 & 40 & 10 & 19832 & 4958 & 5685 \\
\hline
\end{tabular}

Table 1. Statistics of the benchmark datasets. $|\cdot|$ represents the cardinality of a set. For the definition of the symbols, see Section 2 of this paper. Examples in the datasets are images, and the splits are proposed in [7].

$$
\begin{aligned}
& L_{1}\left(f_{i}, s_{i} ; \theta\right) \\
& =\frac{1}{\left|S_{t r}\right|-1} \sum_{s \in S_{t r} \backslash\left\{s_{i}\right\}} c^{2}\left(f_{i}, s ; \theta\right)+\left[c\left(f_{i}, s_{i} ; \theta\right)-1\right]^{2}
\end{aligned}
$$

The SB loss is formulated correspondingly as:

$$
\begin{aligned}
& L_{2}\left(f_{i}, s_{i}, s_{j} ; \theta\right) \\
& =\frac{\sum_{s \in S_{t r} \backslash\left\{s_{i}, s_{j}\right\}} c^{2}\left(f_{i}, s ; \theta\right)}{\left|S_{t r}\right|-\left|\left\{s_{i}, s_{j}\right\}\right|}+\left[c\left(f_{i}, s_{j} ; \theta\right)-1\right]^{2}
\end{aligned}
$$

\subsection{Semantic similarities}

When using SB to improve GZSL methods with compatibility metric learning, it is necessary to borrow the most similar semantic vector to each training semantic vector in the training set, which requires the calculation of the semantic similarity. Thanks to the process of determining the similarity in SB independent of the objective, in the case that the training semantics are equaldimensional vectors of attributes, we can use negative MAE as the semantic similarity to make the semantic comparison more precise. Therefore, the function for seeking similar semantics can be formulated as:

$$
C\left(s_{j}\right)=\underset{s \in S_{t r}}{\operatorname{argmin}}\left\|s-s_{j}\right\|_{1}
$$

\subsection{Recognition}

By minimizing Eq. 5, we can obtain the compatibilities between features and semantics. Based on the learned compatibility function, a multi-class classifier $M: F \rightarrow S$ that achieves the goal of GZSL can be formulated as follows:

$$
M(f)=\underset{s \in S}{\operatorname{argmax}} c(f, s)
$$

where $f \in F$. Then the class corresponding to $M(f)$ is what we want.

\section{EXPERIMENTS}

\subsection{Evaluation protocol and implementation details}

In order to evaluate SB, Caltech-UCSD Birds-200-2011 (CUB) [24] is selected as the representative of fine-grained benchmark datasets, and Animals with Attributes 1 (AWA1) [3] as the representative of coarse-grained benchmark datasets, which are summarized in Table 1. The splits, semantics and evaluation metrics used in the comparison are proposed in [7], where semantics are class-level attributes. Following [7, 21, 25], example features are the 2048dimensional top pooling units of a ResNet-101 pre-trained on

\begin{tabular}{l|ccc|ccc}
\hline \multirow{2}{*}{ Metric } & \multicolumn{3}{|c|}{ CUB } & \multicolumn{3}{c}{ AWA1 } \\
\cline { 2 - 7 } & $\mathbf{u}$ & $\mathbf{s}$ & $\mathbf{h}$ & $\mathbf{u}$ & $\mathbf{s}$ & $\mathbf{h}$ \\
\hline \hline -MSE & 27.8 & $\mathbf{6 0 . 2}$ & 38.0 & 17.6 & 87.6 & 29.3 \\
$\cos$ & 27.8 & 59.3 & 37.8 & 17.3 & 87.2 & 28.9 \\
R & 28.1 & 58.5 & 38.0 & 15.8 & $\mathbf{8 8 . 5}$ & 26.8 \\
\hline -MAE & $\mathbf{2 9 . 1}$ & 59.8 & $\mathbf{3 9 . 1}$ & $\mathbf{1 9 . 3}$ & 86.2 & $\mathbf{3 1 . 5}$ \\
\hline
\end{tabular}

Table 2. Evaluation of different semantic similarity metrics used in SB.

ImageNet-1K, without any preprocessing. Average per-class top-1 accuracies in \% (T-1) are calculated as evaluation scores. The metrics $\mathrm{u}$ and $\mathrm{s}$ are $\mathrm{T}-1$ of unseen and seen classes, respectively, and $\mathrm{h}$ is their harmonic mean [7]. $\mathrm{u}$ reflects the performance of a classifier for unseen classes, $\mathrm{s}$ reflects the performance for seen classes, and $\mathrm{h}$ indicates the comprehensive performance.

The experiments comprehensively evaluate SB with different models. The bilinear mapping [26] is selected as the representative of the linear model, and the MLP combination used in [23] as the representative of the nonlinear model. The combination consists of 2 MLPs with 1 hidden layer, and the numbers of hidden units are hyperparameters. The first MLP maps semantics into prototype features, and the second MLP maps the concatinations of features and prototype features into compatibilities. Each layer has a ReLU activation function, except for the last layer with a sigmoid activation function. The former model is optimized with minibatch SGD while the latter model is optimized with Adam. Hyperparameters are tuned on validation sets.

\subsection{Comparison with strong baselines}

A similarity function related to the objective has to be usable for the training algorithm. For example, when training with gradient descent, the similarity function is required to be differentiable. But benefitting from SB independent of the loss, when comparing the semantics, we can explore more kinds of metrics. Table 2 lists the performance evaluation of different similarity matrics for SB with linear models on the fine-grained and coarse-grained datasets. The first three rows are the negative mean square error (-MSE), cosine similarity (cos), and Ruzicka similarity (R) as strong baselines, and the last row is the negative mean absolute error (-MAE) [27] for the framework in this paper. Each triple in the table comes from the same trained model, whereas different triples come from different trained models.

Comparing $\mathrm{h}$ scores of the baselines, we can see that the best similarity matric on the fine-grained dataset may not be the best on the coarse-grained dataset and the worst has the same observation, and vice versa. It shows that it is beneficial for improving the performance of a classifier to be able to explore more kinds of metrics. We can observe that using MAE can get better results on $h$ and $\mathrm{u}$ than using other metrics, which shows that part of the contribution of SB to the performance improvement of GZSL methods comes from the availability of MAE. We can also notice that MAE is not better than the baselines on all scores. There are situations where its improvement on $\mathrm{h}$ is at the cost of reducing $\mathrm{s}$. It warns us that we cannot always use MAE with the highest $h$ score if a good s score is required for a specific application.

\subsection{Comparison with some present methods}




\begin{tabular}{l|ccc|ccc}
\hline \multirow{2}{*}{ Method } & \multicolumn{3}{|c|}{ CUB } & \multicolumn{3}{c}{ AWA1 } \\
\cline { 2 - 7 } & $\mathbf{u}$ & $\mathbf{s}$ & $\mathbf{h}$ & $\mathbf{u}$ & $\mathbf{s}$ & $\mathbf{h}$ \\
\hline DAP [3] & 1.7 & 67.9 & 3.3 & 0.0 & $\mathbf{8 8 . 7}$ & 0.0 \\
IAP [3] & 0.2 & $\mathbf{7 2 . 8}$ & 0.4 & 2.1 & 78.2 & 4.1 \\
CONSE [17] & 1.6 & 72.2 & 3.1 & 0.4 & 88.6 & 0.8 \\
CMT [11] & 7.2 & 49.8 & 12.6 & 0.9 & 87.6 & 1.8 \\
SSE [16] & 8.5 & 46.9 & 14.4 & 7.0 & 80.5 & 12.9 \\
LATEM [10] & 15.2 & 57.3 & 24.0 & 7.3 & 71.7 & 13.3 \\
ALE [13] & 23.7 & 62.8 & 34.4 & 16.8 & 76.1 & 27.5 \\
DEVISE [14] & 23.8 & 53.0 & 32.8 & 13.4 & 68.7 & 22.4 \\
SJE [9] & 23.5 & 59.2 & 33.6 & 11.3 & 74.6 & 19.6 \\
ESZSL [28] & 12.6 & 63.8 & 21.0 & 6.6 & 75.6 & 12.1 \\
SYNC [15] & 11.5 & 70.9 & 19.8 & 8.9 & 87.3 & 16.2 \\
SAE [18] & 7.8 & 54.0 & 13.6 & 1.8 & 77.1 & 3.5 \\
\hline Proposed & $\mathbf{2 9 . 1}$ & 59.8 & $\mathbf{3 9 . 1}$ & $\mathbf{1 9 . 3}$ & 86.2 & $\mathbf{3 1 . 5}$ \\
\hline \hline GAZSL [20] & 31.7 & 61.3 & 41.8 & 29.6 & 84.2 & 43.8 \\
FGN [21] & $\mathbf{4 3 . 7}$ & 57.7 & 49.7 & $\mathbf{5 7 . 9}$ & 61.4 & $\mathbf{5 9 . 6}$ \\
RN [23] & 38.1 & 61.4 & 47.0 & 31.4 & $\mathbf{9 1 . 3}$ & 46.7 \\
\hline Proposed & 41.7 & $\mathbf{6 4 . 2}$ & $\mathbf{5 0 . 6}$ & 33.0 & 87.2 & 47.8 \\
\hline
\end{tabular}

Table 3. Comparison with some present methods on the benchmarks from [7]. Other results are taken from the papers.

There have been proposed methods that can be used to solve the GZSL problem to some extent. Compared with them, we can see that SB can build new force for GZSL. In Table 3, a linear model and a nonlinear model trained with SB are compared with some present GZSL methods. The results of linear models are listed in the upper half of the table, and the results of nonlinear models in the lower half.

Among linear models, it is easy to see that the models trained with SB get the best $h$ and $u$, which shows that they are less biased towards seen classes than those without SB and the comprehensive performance is also improved, as described in the introduction. Among nonlinear models, the model trained with SB obtains the best $\mathrm{h}$ on the fine-grained dataset. It needs to be added that, unlike all other models in the table, which are trained under the CIII training setting where testing data are invisible, [20] and [21] use testing semantics to synthesize examples for unseen classes so as to learn the final classifier. Therefore, it is impossible for them to be used under CIII. The comparison with them is added to the table to improve completeness. In fact, the use of SB in a synthesizing method with compatibility metric learning can be a future study, where SB will be used in non-CIII training settings.

\subsection{Effectiveness}

In order to verify the effectiveness of $\mathrm{SB}$, an ablation study is conducted here. Table 4 demonstrates the comparison of models trained with and without SB. It can be seen that SB improves $h$ and $\mathrm{u}$ of both linear and non-linear models on both fine-grained and coarse-grained datasets, and in some cases also improves s, thanks to the more accurately modeled relationship between the semantics of unseen and seen classes with SB.

\begin{tabular}{l|ccc|ccc}
\hline \multirow{2}{*}{ Model } & \multicolumn{3}{|c|}{ CUB } & \multicolumn{3}{c}{ AWA1 } \\
\cline { 2 - 7 } & u & s & h & u & s & h \\
\hline \hline linear & 27.2 & $\mathbf{5 9 . 9}$ & 37.4 & 18.0 & 84.3 & 29.6 \\
linear+SB & $\mathbf{2 9 . 1}$ & 59.8 & $\mathbf{3 9 . 1}$ & $\mathbf{1 9 . 3}$ & $\mathbf{8 6 . 2}$ & $\mathbf{3 1 . 5}$ \\
\hline \hline nonlinear & 40.0 & 63.0 & 48.9 & 32.5 & $\mathbf{8 7 . 9}$ & 47.4 \\
nonlinear+SB & $\mathbf{4 1 . 7}$ & $\mathbf{6 4 . 2}$ & $\mathbf{5 0 . 6}$ & $\mathbf{3 3 . 0}$ & 87.2 & $\mathbf{4 7 . 8}$ \\
\hline
\end{tabular}

Table 4. Comparison of models trained with and without SB.

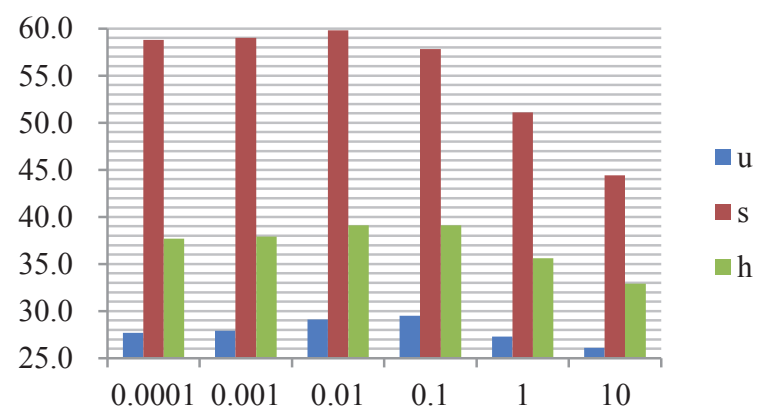

Fig. 2. Analysis of the influence of the value of $\boldsymbol{\alpha}$ on (from left to right) $\mathrm{u}, \mathrm{s}$ and $\mathrm{h}$ scores of a linear model on CUB.

\subsection{Effect}

The effect of SB on the original method is influenced by $\alpha$ in Eq. 5 . By evaluating the models trained with different $\alpha$, we can be more clear about the way SB takes effect. For this purpose, a set of linear models are optimized with different $\alpha$ on CUB. Fig. 2 shows the evaluation results of 6 representative values of $\alpha$. Combined with Table 4, it can be seen that the models are worse than those trained without $\mathrm{SB}$ when $\alpha \geq 1$. It is expected because the compatibility between each feature and its semantically similar semantic vector is learned additionally with SB, so that the relationship between the semantics of unseen and seen classes is modeled more accurately, which improves the performance of the GZSL classifier, but when each compatibility of this kind is greater than or equal to the compatibility between the feature and its corresponding semantic vector, the relationship modeling becomes worse. In addition, we can observe that the model obtains the best h and s when $\alpha=0.01$ and the best $\mathrm{h}$ and $\mathrm{u}$ when $\alpha=0.1$. On the both sides, the performance of the model decreases. It shows again that modeling a too large or too small compatibility between each feature and its semantically similar semantic vector will lead to inaccuracy, thereby reducing the improvement of the original method by SB.

\section{CONCLUSION}

In this work, semantic borrowing based on MAE is proposed to improve GZSL methods with compatibility metric learning under CIII. Extensive evaluation of representative models trained on representative GZSL benchmark datasets with the proposed method has shown that it can improve the performance of generalized zero-shot classification.

Acknowledgements. The numerical calculations reported in the paper were performed in Google Colaboratory. Thanks to Google for its contribution to the development of AI. 


\section{REFERENCES}

[1] X. Zhu, D. Anguelov, and D. Ramanan, "Capturing long-tail distributions of object subcategories," in Proc. Comput. Vis. Pattern Recognit., pp. 915-922, 2014.

[2] W. Wang, V.W. Zheng, H. Yu, and C. Miao. "A survey of zero-shot learning: settings, methods, and applications," ACM Trans. Int.. Syst. Techn., Vol. 10, No. 2, Art. 13 , pp. 45, Jan. 2019.

[3] C.H. Lampert, H. Nickisch, and S. Harmeling, "Learning to detect unseen object classes by between-class attribute transfer," in Proc. of the IEEE Conference on Computer Vision and Pattern Recognition, 2009, pp. 951-958.

[4] H. Larochelle, D. Erhan, and Y. Bengio, "Zero-data learning of new tasks," in Proceedings of the Twenty-Third AAAI Conference on Artificial Intelligence, 2008, pp. 646-651.

[5] M. Palatucci, D. Pomerleau, G. Hinton, and T.M. Mitchell, "Zero-shot learning with semantic output codes," in $A d v$. in Neur. Inf. Proc. Syst. 22: 23rd Annual Conference on Neural Information Processing Systems, 2009, pp. 1410-1418.

[6] W.-L. Chao, S. Changpinyo, B. Gong, and F. Sha, "An empirical study and analysis of generalized zero-shot learning for object recognition in the wild," in European Conference on Computer Vision, 2016, pp. 52-68.

[7] Y. Xian, C.H. Lampert, B. Schiele, and Z. Akata, "Zero-shot learning - a comprehensive evaluation of the good, the bad and the ugly," arXiv preprint arXiv:1707.00600v3, pp. 22512265, 2017.

[8] Z. Akata, F. Perronnin, Z. Harchaoui, and C. Schmid, "Label-embedding for image classification," IEEE Trans. on Patt. Anal. and Mach. Intell., 38, 7, pp. 1425-1438, 2016.

[9] Z. Akata, S. Reed, D. Walter, H. Lee, and B. Schiele, "Evaluation of output embeddings for fine-grained image classification," in Proc. of the IEEE Conf. on Computer Vision and Pattern Recognition, 2015, pp. 2927-2936.

[10] Y. Xian, Z. Akata, G. Sharma, Q. Nguyen, M. Hein, and B. Schiele, "Latent embeddings for zero-shot classification," in Proc. of the IEEE Conf. on Comp. Vis. and Patt. Rec., 2016, pp. 69-77.

[11] R. Socher, M. Ganjoo, C.D. Manning, and A.Y. Ng, "Zeroshot learning through cross-modal transfer," in Adv. in Neur. Inform. Processing Systems 26: 27th Annual Conference on Neural Information Processing Systems, 2013, pp. 935-943.

[12] S. Reed, Z. Akata, H. Lee, and B. Schiele, "Learning deep representations of fine-grained visual descriptions," in Proc. of the IEEE Conf. on Comp. Vis. and Patt. Recognition, 2016, pp. 49-58.

[13] Z. Akata, F. Perronnin, Z. Harchaoui, and C. Schmid, "Label-embedding for attribute-based classification," in Proc. of the IEEE Conf. on Comp. Vis. and Pattern Recognition, 2013, pp. 819-826.

[14] A. Frome, G.S. Corrado, J. Shlens, S. Bengio, J. Dean, M.'A. Ranzato, and T. Mikolov, "DeViSE: A deep visual-semantic embedding model," in Adv. in Neur. Inf. Proc. Syst. 26: 27th Ann. Conf. on Neur. Inf. Proc. Systems, 2013, pp. 2121-2129.

[15] S. Changpinyo, W.-L. Chao, B. Gong, and F. Sha, "Synthesized classifiers for zero-shot learning," in Proc. of the IEEE Conf. on Comp. Vis. and Patt. Rec., 2016, pp. 5327-5336.

[16] Z. Zhang and V. Saligrama, "Zero-shot learning via semantic similarity embedding," in Proc. of the IEEE International Conference on Computer Vision, 2015, pp. 4166-4174.

[17] M. Norouzi, T. Mikolov, S. Bengio, Y. Singer, J. Shlens, A. Frome, G.S. Corrado, and J. Dean, "Zero-shot learning by convex combination of semantic embeddings," in Int. Conference on Learning Representations, 2014, pp. .

[18] E. Kodirov, T. Xiang, and S. Gong, "Semantic autoencoder for zero-shot learning," in Proceedings of the IEEE Conference on Computer Vision and Pattern Recognition, 2017, pp. 4447-4456.

[19] Jiang, Huajie, R. Wang, S. Shan, and X. Chen. "Adaptive metric learning for zero-shot recognition," IEEE Signal Processing Letters, 26, no. 9, pp. 1270-1274, 2019.

[20] Y. Zhu, M. Elhoseiny, B. Liu, X. Peng, and A. Elgammal, "A generative adversarial approach for zero-shot learning from noisy texts," in Proceedings of the IEEE Conference on Computer Vision and Pattern Recognition, 2018, pp. 10041013.

[21] Y. Xian, T. Lorenz, B. Schiele, and Z. Akata, "Feature generating networks for zero-shot learning," In Proc. of the IEEE Conf. on Comp. Vis. and Pattern Recognition, 2018, pp. $5542-5551$

[22] Y. Fu, T. M. Hospedales, T. Xiang, and S. Gong, "Transductive multi-view zero-shot learning," IEEE Trans. Pattern Anal. Mach. Intell., vol. 37, no. 11, pp. 2332-2345, Nov. 2015

[23] F. Sung, Y. Yang, X. Lin, T. Xiang, P.H.S. Torr, and T.M. Hospedales, "Learning to compare: relation network for fewshot learning," in Proc. Conf. Comput. Vis. Pattern Recognit., 2018, pp. 1199-1208.

[24] C. Wah, S. Branson, P. Welinder, P. Perona, and S. Belongie, "The caltech-ucsd birds-200-2011 dataset," Technical Report CNS-TR-2011-001, California Institute of Technology, 2011.

[25] M. Bucher, S. Herbin, and F. Jurie, "Generating visual representations for zero-shot classification," in IEEE Int. Conf. on Computer Vision Workshops: Transferring and Adapting Source Knowledge in Computer Vision, 2017, pp. 2666-2673.

[26] Sariyildiz, M. Bulent, and R.G. Cinbis. "Gradient matching generative networks for zero-shot learning," in Proc. of the IEEE Conf. on Comp. Vis. and Pattern Recognition, 2019, pp. 2168-2178.

[27] Deza, M. Marie, and E. Deza, Encyclopedia of distances, Springer, Berlin, Heidelberg, 2009.

[28] B. Romera-Paredes and P.H.S. Torr, "An embarrassingly simple approach to zero-shot learning," in Proc. of the 32nd Int. Conference on Machine Learning, 2015, pp. 2152-2161. 